\title{
L'hydrolyse du lactose et la fabrication de lait chocolaté
}

\author{
par \\ K. VANDAMME et R. DELBEKE*
}

INTRODUCTION

Dans la fabrication de lait chocolaté, on ajoute souvent 5 à 8 p. 100 de saccharose afin de conférer au produit le goût sucré désiré. Cette dose supplémentaire de sucre peut être limitée si on met le potentiel sucrant propre du lait mieux à profit en hydrolysant le lactose. Ceci est un moyen de limiter la surconsommation d'hydrates de carbone.

Un second aspect justifiant l'hydrolyse est l'intolérance à l'égard $\mathrm{du}$ lactose. Une insuffisance de la production de lactase ( $\beta$ galactosidase) dans l'intestin grêle peut causer des troubles intestinaux. La paroi intestinale ne peut, en effet, résorber le lactose qu'après hydrolyse enzymatique de ce sucre en glucose et en galactose. L'intolérance à l'égard du lactose varie d'une personne à l'autre et est plus fréquente chez certaines races humaines.

Au cours des essais, le lactose a d'abord été hydrolysé en cuvées de lait par une méthode de courte durée, à l'aide de préparations d'enzymes employées à des doses relativement élevées.

On a examiné ensuite les possibilités d'hydrolyse de longue durée du lactose à l'aide de petites doses d'enzyme, l'enzyme étant ajoutée en conditions aseptiques à du lait UHT.

Dans aucun des cas le rendement économique nous semblait suffisant pour continuer les essais avec du lait chocolaté.

\section{MATERIELS ET METHODES}

\section{Substrat et conditions des essais}

Les essais en cuvées avec des doses d'enzyme relativement élevées ont été effectués dans les conditions de température et de durée sui-

* Station Laitière de l'Etat, Melle, Centre de Recherches Agronomiques de l'Etat, Gand (Belgique). 
vantes : $5^{\circ} \mathrm{C}-16 \mathrm{~h}, 10^{\circ} \mathrm{C}-16 \mathrm{~h}, 30^{\circ} \mathrm{C}-3 \mathrm{~h}$ et $30^{\circ} \mathrm{C}-6 \mathrm{~h}$. Ces combinaisons ont été choisies d'après les possibilités de la ligne de production pour lait chocolaté d'une laiterie déterminée. Le substrat utilisé était du lait pasteurisé $\left(15 \mathrm{~s}-73^{\circ} \mathrm{C}\right)$ avec $1,5 \mathrm{p} .100$ de matière grasse.

Les essais en conditions aseptiques, avec de petites doses d'enzyme ont été menés selon la méthode de Dahlqvist et al. [4]. La solution d'enzyme était stérilisée par passage dans un filtre Millipore de 220 $\mathrm{nm}$, puis ajoutée en conditions d'asepsie à un lait UHT (1,5 p. 100 de matière grasse) figurant comme substrat.

\section{Enzymes}

Les enzymes ci-après ont été utilisées pour les essais en conditions non aseptiques, d'hydrolyse de courte durée en cuvées, par des doses d'enzyme relativement élevées :

- Maxilact L 2000 et Maxilact LX 5000 de la firme Gist-Brocades N.V., Delft (Pays-Bas) [6] ;

- Novo 1500 L de la firme Novo Industri A.S., Bagsvaerd (Danemark) [8] ;

- Godo YNL de la firme Godo Shusei, Tokyo (Japon) [7].

Dans les essais d'hydrolyse de longue durée, dans les emballages, par une petite dose d'enzyme stérile, celle-ci était la Maxilact LX 5000 précitée.

\section{Détermination du degré d'hydrolyse}

Les sucres (lactose, galactose et glucose) ont été déterminés par la méthode enzymatique décrite par Boehringer [1]. Le degré d'hydrolyse a été calculé d'après les quantités de sucre présentes avant et après l'hydrolyse du lactose.

Pour inactiver la lactase les échantillons étaient chauffés pendant $15 \mathrm{~min}$ à $75^{\circ} \mathrm{C}$. Ils étaient ensuite clarifiés au moyen de solutions de Carrez.

\section{RESULTATS ET DISCUSSION}

\section{Résultats des essais}

\subsection{Hydrolyse non aseptique a l'Aide de doses d'enzyme ÉlevéEs}

Si l'hydrolyse n'est pas effectuée en conditions d'asepsie, il est préférable de limiter sa durée de façon à éviter les risques bactériologiques. Des doses d'enzyme relativement élevées sont dès lors nécessaires pour procurer un degré d'hydrolyse satisfaisant. Une dose élevée est d'ailleurs généralement nécessaire pour respecter le schéma de temps et de température d'une ligne de production déterminée. 
TABLEAU 1

Doses d'enzyme à employer pour obtenir, dans différentes conditions, une hydrolyse à 75 p. 100 du lactose du lait Coût des doses en FB

\begin{tabular}{|c|c|c|c|c|c|c|c|c|c|}
\hline \multirow{2}{*}{\multicolumn{2}{|c|}{$\begin{array}{l}\text { Enzyme } \\
\text { type et coût }\end{array}$}} & \multicolumn{8}{|c|}{$\begin{array}{l}\text { Dose d'enzyme utilisée par litre de lait } \\
\text { Coût de la dose en FB }\end{array}$} \\
\hline & & \multicolumn{2}{|c|}{$5^{\circ} \mathrm{C}-16 \mathrm{~h}$} & \multicolumn{2}{|c|}{$10^{\circ} \mathrm{C}-16 \mathrm{~h}$} & \multicolumn{2}{|c|}{$30^{\circ} \mathrm{C}-3 \mathrm{~h}$} & \multicolumn{2}{|c|}{$30^{\circ} \mathrm{C}-6 \mathrm{~h}$} \\
\hline Enzyme & $\mathrm{FB} / \mathrm{kg}$ & $\mathrm{mg} / \mathrm{l}$ & FB & $\mathrm{mg} / \mathrm{l}$ & FB & $\mathrm{mg} / \mathrm{l}$ & FB & $\mathrm{mg} / \mathrm{l}$ & FB \\
\hline Maxilact L 2000 & 690 & 1000 & 0,69 & 850 & 0,59 & 1000 & 0,69 & 850 & 0,59 \\
\hline Maxilact LX 5000 & 5500 & 350 & 1,93 & 225 & 1,23 & 350 & 1,93 & 200 & 1,10 \\
\hline Novo $1500 \mathrm{~L}$ & 675 & 3300 & 2,23 & 2400 & 1,62 & 3300 & 2,23 & 2200 & 1,49 \\
\hline Godo YNL & 4500 & 500 & 2,25 & 390 & 1,75 & 350 & 1,57 & 285 & 1,28 \\
\hline
\end{tabular}


Le tableau 1 indique pour les différentes enzymes essayées la dose à employer pour obtenir, dans les conditions de l'essai, une hydrolyse à 75 p. 100 du lactose du lait. Le même tableau indique aussi le coût de cette dose. Tendre à obtenir une hydrolyse totale dans un temps limité ne peut se justifier, parce que celle-ci nécessite des doses d'enzyme très élevées, du fait de l'inhibition en retour par le galactose formé au cours de l'opération.

D'après le tableau 1 , les combinaisons $10^{\circ} \mathrm{C}-16 \mathrm{~h}$ et $30^{\circ} \mathrm{C}-6 \mathrm{~h}$ avec Maxilact L 2000 coûtent les moins cher (0,59 FB). Elles présentent néanmoins plus de risques bactériologiques que la combinaison $5^{\circ} \mathrm{C}$ $16 \mathrm{~h}$ pour laquelle le coût s'élève avec Maxilact L 2000 à 0,69 FB, montant légèrement supérieur.

Selon certaines données bibliographiques, $\mathrm{Na}$ et $\mathrm{Ca}$ exercent un effet inhibiteur, tandis que $\mathrm{K}, \mathrm{Mg}$ et $\mathrm{Mn}$ ont un effet stimulant sur l'activité enzymatique. Pour étudier l'influence de ces activateurs, on a opéré sur du lait pasteurisé $\left(15 \mathrm{~s}-73^{\circ} \mathrm{C}\right)$ additionné de $500 \mathrm{mg}$ de Maxilact L 2000 au litre. La durée et la température d'hydrolyse étaient respectivement de $3 \mathrm{~h}$ et de $30^{\circ} \mathrm{C}$. $\mathrm{K}, \mathrm{Mg}$ et $\mathrm{Mn}$ étaient ajoutés sous forme de chlorures et en doses croissantes.

Le tableau 2 reproduit les valeurs relatives du degré d'hydrolyse atteint, celui obtenu sans additif étant posé égal à 100 p. 100. Ce tableau montre qu'aucune amélioration spectaculaire du rendement ne peut être obtenue. On parvient tout au plus à économiser 25 p. 100 d'enzyme.

Selon Webb et Johnson [11] le lait a déjà au départ des teneurs moyennes en $\mathrm{K}$ de 1410 p.p.m. $\left(36 \times 10^{-3} \mathrm{M}\right)$, en $\mathrm{Mg}$ de 120 p.p.m. $\left(5 \times 10^{-3} \mathbf{M}\right)$ et en $\mathrm{Mn}$ de 0,2 p.p.m. $\left(4 \times 10^{-6} \mathrm{M}\right)$. Pour améliorer de

\section{TABLEAU 2}

Degré d'hydrolyse relatif du lactose obtenu dans le lait par l'addition supplémentaire de doses croissantes de quelques cations

\begin{tabular}{|c|c|c|c|}
\hline \multirow{2}{*}{$\begin{array}{l}\text { Quantité ajoutée en supplément } \\
\text { en mols par litre }\end{array}$} & \multicolumn{3}{|c|}{ Degré d'hydrolyse relatif en p. 100} \\
\hline & K & $\mathrm{Mg}$ & $\mathrm{Mn}$ \\
\hline 0 & 100 & 100 & 100 \\
\hline $10^{-4}$ & 100 & 105 & 106 \\
\hline $5.10^{-4}$ & 100 & 108 & 112 \\
\hline $10^{-3}$ & 102 & 112 & 116 \\
\hline $5.10^{-3}$ & 109 & 117 & 118 \\
\hline $10^{-2}$ & 116 & 126 & 119 \\
\hline $5.10^{-2}$ & 126 & 103 & 92 \\
\hline
\end{tabular}


20 p. 100 le rendement, il faut ajouter $1000 \mathrm{mg} \mathrm{K}, 200 \mathrm{mg}$ Mg ou $550 \mathrm{mg}$ Mn par litre. Ce sont là des doses anormalement élevées, inutilisables dans des boissons lactées telles que le lait chocolaté.

\subsection{Hydrolyse aseptique PAR DE PETITES Doses d'ENZyMe}

On peut limiter la dose d'enzyme en prolongeant la durée de l'hydrolyse. Ceci implique le risque de décomposition bactérienne, qu'il faut éviter en ajoutant, dans des conditions d'asepsie, une enzyme stérile à du lait stérile. L'amélioration éventuelle du rendement financier restera plutôt restreinte, vu qu'il faut tout de même recourir à une quantité supplémentaire de saccharose afin de satisfaire aux préférences de la clientèle. L'ingestion d'hydrates de carbone n'est donc diminuée que dans une mesure assez limitée.

\section{TABLEAU 3}

Degré d'hydrolyse du lactose dans le lait obtenu en conditions stériles au moyen de petites doses d'enzyme au cours d'une conservation de longue durée à des températures différentes

\begin{tabular}{|c|c|c|c|c|c|c|c|c|c|}
\hline \multirow{2}{*}{$\begin{array}{l}\text { Température } \\
\text { de conservation } \\
\text { en }{ }^{\circ} \mathrm{C}\end{array}$} & \multirow{2}{*}{$\begin{array}{l}\text { Dose d'enzyme } \\
\text { en mg } \\
\text { par litre }\end{array}$} & \multicolumn{8}{|c|}{$\begin{array}{l}\text { Degré d'hydrolyse en p. } 100 \\
\text { Durée de la conservation en jours }\end{array}$} \\
\hline & & 1 & 2 & 4 & 6 & 8 & 10 & 15 & 20 \\
\hline 5 & $\begin{array}{r}5 \\
10 \\
20 \\
30 \\
40 \\
50\end{array}$ & $\begin{array}{r}4 \\
10 \\
19 \\
27 \\
38 \\
45\end{array}$ & $\begin{array}{r}7 \\
13 \\
26 \\
37 \\
48 \\
55\end{array}$ & $\begin{array}{l}12 \\
20 \\
38 \\
50 \\
61 \\
70\end{array}$ & $\begin{array}{l}17 \\
28 \\
48 \\
59 \\
69 \\
76\end{array}$ & $\begin{array}{l}22 \\
35 \\
56 \\
67 \\
76 \\
81\end{array}$ & $\begin{array}{l}27 \\
44 \\
64 \\
75 \\
82 \\
86\end{array}$ & $\begin{array}{l}37 \\
58 \\
74 \\
84 \\
88 \\
91\end{array}$ & $\begin{array}{l}41 \\
64 \\
80 \\
88 \\
91 \\
92\end{array}$ \\
\hline 10 & $\begin{array}{r}5 \\
10 \\
20 \\
30 \\
40 \\
50\end{array}$ & $\begin{array}{r}5 \\
16 \\
26 \\
36 \\
48 \\
54\end{array}$ & $\begin{array}{l}10 \\
24 \\
35 \\
45 \\
54 \\
62\end{array}$ & $\begin{array}{l}15 \\
35 \\
42 \\
56 \\
63 \\
72\end{array}$ & $\begin{array}{l}20 \\
43 \\
53 \\
64 \\
72 \\
78\end{array}$ & $\begin{array}{l}25 \\
48 \\
60 \\
70 \\
78 \\
84\end{array}$ & $\begin{array}{l}31 \\
52 \\
66 \\
75 \\
84 \\
88\end{array}$ & $\begin{array}{l}42 \\
60 \\
76 \\
84 \\
90 \\
92\end{array}$ & $\begin{array}{l}48 \\
66 \\
79 \\
86 \\
92 \\
93\end{array}$ \\
\hline 20 & $\begin{array}{r}5 \\
10 \\
20 \\
30 \\
40 \\
50\end{array}$ & $\begin{array}{l}20 \\
31 \\
48 \\
57 \\
67 \\
75\end{array}$ & $\begin{array}{l}25 \\
39 \\
57 \\
66 \\
75 \\
81\end{array}$ & $\begin{array}{l}34 \\
50 \\
68 \\
76 \\
84 \\
88\end{array}$ & $\begin{array}{l}41 \\
59 \\
78 \\
84 \\
90 \\
93\end{array}$ & $\begin{array}{l}47 \\
66 \\
83 \\
88 \\
92 \\
95\end{array}$ & $\begin{array}{l}52 \\
71 \\
88 \\
91 \\
94 \\
96\end{array}$ & $\begin{array}{l}64 \\
79 \\
91 \\
94 \\
96 \\
97\end{array}$ & \\
\hline
\end{tabular}


En outre, on ne peut pas perdre de vue que selon cette méthode on ne cherche pas à détruire l'enzyme après un temps déterminé.

Le tableau 3 indique le degré d'hydrolyse obtenu au moyen de doses croissantes, de 5 à $50 \mathrm{mg}$, d'enzyme Maxilact LX 5000 par litre de lait et à des températures de conservation de 5,10 et $20^{\circ} \mathrm{C}$. A ces températures, on a obtenu déjà au bout de 10 jours, avec $20 \mathrm{mg}$ d'enzyme, des degrés d'hydrolyse de respectivement 64,66 et 88 p. 100 .

A $20^{\circ} \mathrm{C}$, on a obtenu au bout de 5 jours, avec $20 \mathrm{mg}$ d'enzyme par litre, une hydrolyse à 75 p. 100 . A $5500 \mathrm{FB} / \mathrm{kg}$, les $20 \mathrm{mg}$ d'enzyme coûtent 0,110 FB.

\section{Aspects économiques}

\subsection{Hydrolyse nON ASEPTique a L'Aide DE DOSES D'ENZyme ÉLEVÉES}

Le tableau 4 indique le pouvoir sucrant relatif des différents sucres d'après les données de Bus et al. [3].

En admettant que le lait a une teneur en lactose de $48 \mathrm{~g} / \mathrm{l}$ et que la fabrication classique de lait chocolaté utilise $70 \mathrm{~g}$ de saccharose par litre de lait afin de satisfaire aux préférences de la clientèle, il découle des données du tableau 4 que, dans ce cas, le pouvoir sucrant relatif est approximativement de $(0,48 \times 39)+(0,70 \times 100)=88,72$.

Le tableau 5 indique l'économie de saccharose maximale pour un degré d'hydrolyse croissant et un pouvoir sucrant relatif et désiré de 88,72 . D'après ce tableau l'économie maximale de sucre s'élève à 15,44 g pour une hydrolyse à 100 p. 100 du lactose. A 75 p. 100 d'hydro-

\section{TABLEAU 4}

Pouvoir sucrant relatif pour différents sucres. Solutions aqueuses à 10 p. 100

\begin{tabular}{l|c}
\hline \multicolumn{1}{c|}{ Sucre } & Pouvoir sucrant relatif \\
& \\
Saccharose & 100 \\
Lactose & 39 \\
Glucose & 72 \\
Galactose & 63 \\
Fructose & 114 \\
Lactose hydrolysé & 68 \\
& \\
\end{tabular}

Tableau d'après les données de Bus et al. [3]. 
TABLEAU 5

L'économie en saccharose pour un degré d'hydrolyse croissant du lactose du lait*

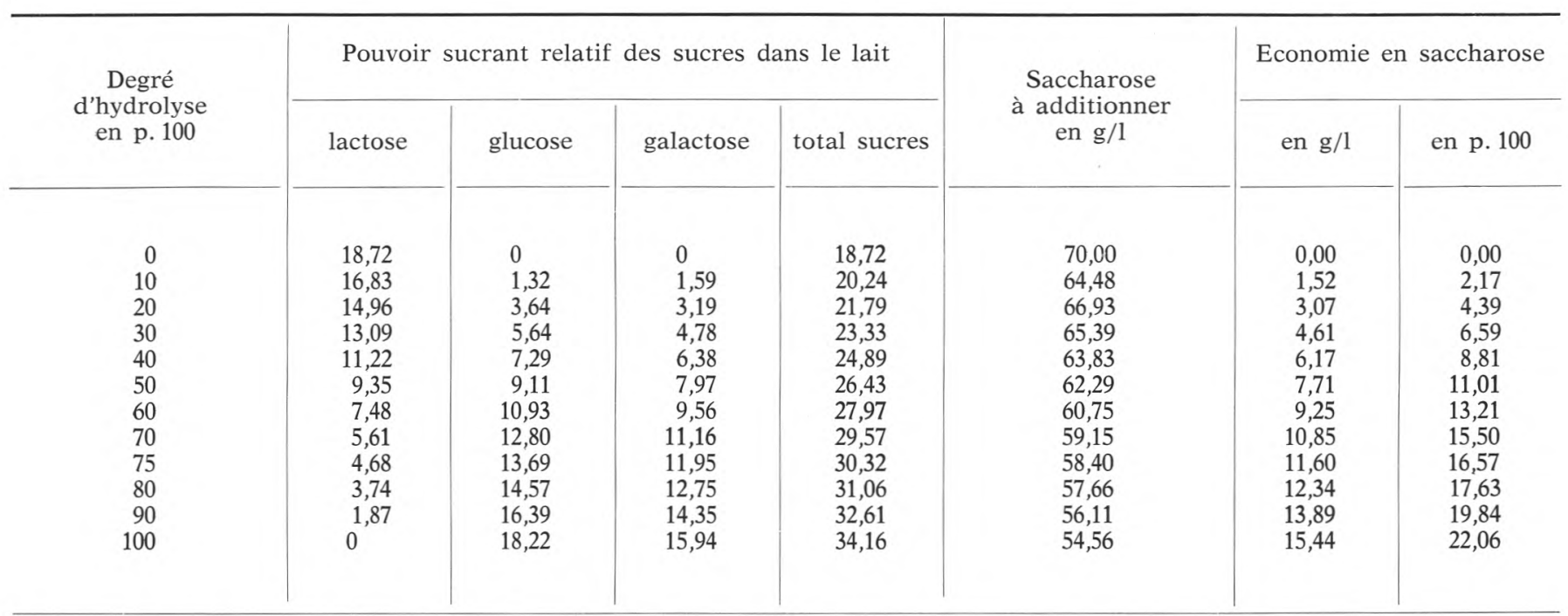

* Bases de calcul :

- lait avec $48 \mathrm{~g}$ de lactose par litre et additionné de $70 \mathrm{~g}$ de saccharose par litre ;

- le pouvoir sucrant relatif désiré est de $(0,48 \times 39)+(0,70 \times 100)=88,72$. 
lyse elle serait de 11,60 g/1. A 21 FB le kilogramme de saccharose cette quantité de $11,60 \mathrm{~g}$ coûte $0,244 \mathrm{FB}$.

D'après le tableau 1 la dépense en enzyme par litre de lait traité s'élève, pour une hydrolyse du lactose à 75 p. 100, dans le cas le moins cher à 0,587 FB $(0,59)$. La dépense en enzyme est donc supérieure de $0,343 \mathrm{FB}$ au montant de $0,244 \mathrm{FB}$ correspondant à l'économie de sucre.

Le fait que le pouvoir sucrant dans le lait peut différer du pouvoir sucrant dans une solution aqueuse ne modifiera guère ces résultats. En outre la rentabilité sera encore plus faible du fait de la formation d'autres oligo-saccharides au cours de l'hydrolyse, ceux-là n'étant pas sucrants. Selon Toba [10], ces oligo-saccharides sont produits par l'effet de transgalactosidase de la lactase. Selon Burvall [2], la teneur en ces oligo-saccharides serait pour du lait d'au moins 5 p. 100 de la teneur totale en sucres à un degré d'hydrolyse de 75 p. 100 .

Enfin, il faut aussi tenir compte, en plus du coût de la dose d'enzyme ajoutée, des frais d'investissement et de fonctionnement supplémentaires.

\subsection{HydRolyse ASEPTIOUE PAR DE PETITES DOSES D'ENZyME}

Selon le tableau 3 on obtient à $20^{\circ} \mathrm{C}$ au bout de 5 jours avec $20 \mathrm{mg}$ d'enzyme Maxilact LX 5000 par litre de lait, un degré d'hydrolyse de 75 p. 100 (78 p. 100 en 6 jours). A 5500 FB/kg, les 20 mg d'enzyme coûtent $0,110 \mathrm{FB}$.

A 75 p. 100 d'hydrolyse on peut selon le tableau 5 économiser $11,60 \mathrm{~g}$ de saccharose par litre de lait dont le coût s'élève à 0,244 FB. La dépense en enzyme est donc, dans ce cas, inférieure de 0,134 FB au montant correspondant à l'économie de sucre. En utilisant $44 \mathrm{mg}$ d'enzyme Maxilact LX 5000 par litre de lait, la dépense en enzyme représenterait un montant équivalant à l'économie de sucre.

Il faut évidemment aussi tenir compte des frais d'investissement et de fonctionnement supplémentaires. La méthode exige un soutirage aseptique, avec dosage de l'enzyme stérile dans le lait chocolaté stérilisé. La firme Tetra Pak [9] offre déjà dans son programme de fabrication l'équipement permettant d'appliquer cette méthode.

Tenant compte de la différence minime entre la dépense en enzyme et l'économie de sucre mentionnée ci-dessus, et les exigences en équipement de la méthode, nous avons estimé que le rendement économique était trop faible pour continuer nos recherches sur l'hydrolyse du lactose dans un substrat de lait chocolaté et déjà sucré.

\section{Enzymes fixées et ultrafiltration}

L'hydrolyse du lactose par des enzymes fixées se révèle provisoirement inappropriée pour le lait et dans la littérature récente on ne trouve pas d'exemples d'installations industrielles traitant le lait selon cette méthode. 
Une laiterie en possession d'une installation d'ultrafiltration servant à la fabrication de concentrés de protéines dispose d'un perméat à bas prix et peut penser à remplacer partiellement le saccharose par un sirop de lactose hydrolysé, préparé en partant de ce perméat.

Il faut $200 \mathrm{mg}$ de Maxilact L 2000 (coût 0,138 FB) pour hydrolyser 75 p. 100 du lactose dans 11 d'un tel perméat en 20 h et à une température de $10^{\circ} \mathrm{C}$. Dans les mêmes conditions il ne faut que $75 \mathrm{mg}$ (coût $0,052 \mathrm{FB}$ ) pour une hydrolyse à 75 p. 100 dans un perméat purifié (résultats d'essais pas encore publiés). Selon Delbeke [5] il ne faut que $14,2 \mathrm{~g}$ de produits chimiques $(8,4 \mathrm{~g} \mathrm{NaOH}$ et $5,8 \mathrm{~g} \mathrm{HCl})$ par litre de perméat pour obtenir avec des échangeurs d'ions une solution de lactose purifiée à 99,75 p. 100 .

Une laiterie disposant d'une installation d'ultrafiltration doit de toute façon chercher à valoriser le perméat et il est donc fort probable qu'elle possède en même temps déjà un équipement permettant de le purifier.

En outre on peut facilement traiter un perméat (purifié ou non) avec des enzymes fixées et on peut même utiliser quelques modules d'ultrafiltration pourvus d'enzymes, fixées ou non.

L'aspect économique est donc fort promettant, mais le procédé n'élimine pas la présence du lactose dans le produit final et ne diminuera pas la consommation d'hydrates de carbone vu que le pouvoir sucrant relatif du lactose hydrolysé est plus bas que celui du saccharose. En plus il faudra tenir compte de l'influence d'un tel sirop sur la qualité du produit final et éventuellement des restrictions d'ordre médical.

\section{CONCLUSION}

Le substrat utilisé dans les essais était du lait (1,5 p. 100 de matière grasse) pasteurisé ou stérilisé.

Dans l'hydrolyse de courte durée, en cuvées, en conditions non aseptiques, la dépense en enzyme est supérieure au montant que l'on peut économiser en réduisant l'addition de sucre sans affaiblir le goût sucré. L'addition d'activateurs tels que $\mathrm{K}, \mathrm{Mg}$ et $\mathrm{Mn}$ n'apporte aucune amélioration sensible sous le rapport de la rentabilité financière et nécessite en outre l'addition d'une quantité inacceptablement élevée de ces cations pour stimuler convenablement l'activité enzymatique.

Si l'on veut obtenir un degré d'hydrolyse satisfaisant avec une faible dose d'enzyme, l'hydrolyse doit pouvoir durer plusieurs jours, ce qui implique qu'il faut opérer en conditions aseptiques. Avec une dose de $20 \mathrm{mg}$ d'enzyme Maxilact LX 5000 par litre de lait UHT on obtient à $20^{\circ} \mathrm{C}$ au bout de 5 jours, un degré d'hydrolyse de 75 p. 100 .

Ceci permet, dans le cas étudié, une économie de 11,60 g de saccha- 
rose représentant un montant de $0,244 \mathrm{FB}$, tandis que les $20 \mathrm{mg}$ d'enzyme ne coûtent que $0,110 \mathrm{FB}$. Le gain financier est donc assez minime et la méthode exige un soutirage aseptique, avec dosage de l'enzyme stérile dans le lait chocolaté stérile, ce qui mène à un équipement coûteux. En outre l'enzyme subsiste après traitement, ce qui peut soulever dans certains pays un problème de santé publique.

$\mathrm{Vu}$ l'aspect économique défavorable dans le premier cas (hydrolyse non aseptique) et peu prometteur dans le deuxième cas (hydrolyse aseptique) nous n'avons pas entamé des recherches sur l'hydrolyse du lactose dans un substrat de lait chocolaté et déjà sucré, et sur l'influence du lactose hydrolysé sur la qualité du produit final.

On peut se poser des questions sur l'aspect économique d'autres possibilités.

L'hydrolyse du lactose par des enzymes fixées se révèle provisoirement inappropriée pour le lait.

Une laiterie en possession d'une installation d'ultrafiltration servant à la fabrication de concentrés de protéines dispose d'un perméat à bas prix qu'elle doit valoriser, et peut penser à remplacer partiellement le saccharose par un sirop de lactose hydrolysé, préparé en partant de ce perméat. Des essais avec un tel perméat (résultats pas encore publiés) ont démontré que dans certaines conditions la dépense en enzyme pour une hydrolyse du lactose à 75 p. 100 s'élève à 0,138 FB, et à $0,052 \mathrm{FB}$ pour un perméat purifié (solution de lactose purifiée à 99,75 p. 100) avec des échangeurs d'ions. En outre on peut facilement traiter un perméat (purifié ou non) avec des enzymes fixées et on peut même utiliser quelques modules d'ultrafiltration pourvus d'enzymes, fixées ou non. L'aspect économique est donc fort promettant, mais le procédé n'élimine pas la présence du lactose dans le produit final et ne diminuera pas la consommation d'hydrates de carbone. En plus il faudra tenir compte de l'influence d'un tel sirop sur la qualité du produit final et éventuellement des restrictions d'ordre médical.

\section{Rés u m é}

Tous les essais ont été faits avec du lait (1,5 p. 100 de matière grasse) pasteurisé ou stérilisé (UHT).

Une hydrolyse de courte durée, en cuvées avec du lait pasteurisé, par des quantités d'enzyme relativement élevées, n'est économiquement pas rentable. Indépendamment de l'espèce d'enzyme utilisée, le coût de celle-ci est toujours supérieur à l'économie réalisée en limitant l'addition de sucre. L'emploi d'activateurs tels que $\mathrm{K}, \mathrm{Mg}$ et $\mathbf{M n}$, n'y apporte pas d'amélioration sensible.

Par contre, une hydrolyse de longue durée, dans l'emballage, par addition en conditions d'asepsie de petites doses d'enzyme présente un bilan économique positif mais minime. Dans certaines conditions, 
l'économie en saccharose est de 0,244 FB, tandis que la dépense en enzyme ne s'élève qu'à 0,110 FB.

Vu l'aspect économique défavorable dans le premier cas (hydrolyse non aseptique) et peu promettant dans le deuxième cas (hydrolyse aseptique), nous n'avons pas entamé des recherches sur l'hydrolyse du lactose dans un substrat de lait chocolaté et déjà sucré et sur l'influence du lactose hydrolysé sur la qualité du produit final.

Une laiterie qui possède une installation d'ultrafiltration peut penser à remplacer partiellement le saccharose par un sirop de lactose hydrolysé, préparé en partant du perméat. Des essais avec un tel perméat (résultats pas encore publiés) ont démontré que, dans certaines conditions, la dépense en enzyme pour une hydrolyse du lactose à 75 p. 100 s'élève à $0,138 \mathrm{FB}$, et à $0,052 \mathrm{FB}$ pour un perméat purifié. L'aspect économique est donc fort promettant, mais le procédé n'élimine pas la présence du lactose dans le produit final et ne diminuera pas la consommation d'hydrates de carbone. En plus il faudra tenir compte de l'influence d'un tel sirop sur la qualité du produit final et éventuellement des restrictions d'ordre médical.

\section{S u m m a r y}

\section{LACTOSE HYDROLYSIS AND THE MANUFACTURING OF CHOCOLATE MILK}

In all the experiments pasteurized or sterilized (UHT) milk (1,5 p. 100 fat) was used as a substrate.

A short-time batch hydrolysis of lactose in pasteurized milk with relative large amounts of enzyme is not paying. Independent of the used type of enzyme, the enzyme costs are always higher than the savings realized by the reduction of the sugar addition. The addition of activators as $\mathrm{K}, \mathrm{Mg}$ and $\mathrm{Mn}$ did not improve sufficiently the economic aspect.

A long-time aseptic hydrolysis in packed UHT-milk with small amounts of sterile enzyme additioned in aseptic conditions gave a positive but small economical bilan. Under certain conditions the savings in saccharose were $0.244 \mathrm{BF}$, while the enzyme costs were only $0.110 \mathrm{BF}$.

Taking into account the unfavourable economic aspect in the first case (non-aseptic hydrolysis) and the hardly promising aspect in the second case (aseptic hydrolysis), we did not start a research on the hydrolysis of the lactose in a substrate of sugared chocolate milk, or a research about the influence of the hydrolyzed lactose on the quality of the final product.

A dairy possessing an ultrafiltration plant can try to replace part of the saccharose by a sirop of hydrolyzed lactose prepared with 
the permeate. Experiments with such a permeate (unpublished results) showed that under certain conditions the enzyme costs for hydrolyzing $75 \mathrm{p} .100$ of the lactose in such a permeate were only $0.138 \mathrm{BF}$ and $0.052 \mathrm{BF}$ if the permeate was purified before hydrolysis. So the economic aspect is very promising, but this way of processing does not eliminate the presence of lactose in the final product and does not lower the consumption of carbohydrates. Besides, one must taken into account the influence of such a sirop on the quality of the final product and the eventuality of medical restrictions.

Reçu pour publication en décembre 1980.

\section{Bibliographie}

[1] Boehringer (Mannheim) (1979). - Enzymatic analysis for food chemistry, 28 and 32 .

[2] Burvall (A.) (1978). - Lactose hydrolysis: nutritional and technological problems. Dissertation, Department of applied nutrition, Chemical Centre, University of Lund, Sweden.

[3] Bus (W. C.), Reuvekamp (H.) \& Swinkels (J.) (1977). - Natuurlijke zoetmiddelen als konkurrenten van saccharose. Glucose informatie $11 \mathrm{nr} 1,3-18$.

[4] Dahlovist (A.), Asp (N. G.), Burvall (A.) and Rausing (H.) (1977). - Hydrolysis of lactose in milk and whey with minute amounts of lactase. J. Dairy Res., 44, 541.

[5] DelbeKe (R.) (1979). - Purification of an ultrafiltration permeate with adsorbent and ion-exchange resins. Neth. Milk Dairy J., 33, 181-192.

[6] Gist-Brocades (N. V.) (1980). - Technical data sheet, Maxilact L 2000 and Maxilact LX 5000.

[7] Godo ShuseI (1979). - Technical data sheet, Godo YNL (lactase).

[8] Novo Industri A.S. (1979). - Application of Yeast Lactases: a Review.

[9] Tetra PAK (1979). - Système Tetra Lacta.

[10] Това (T.) and Adachi (S.) (1978). - Hydrolysis of lactose by microbial $\beta$ galactosidases. Formation of oligosaccharides with special references to 2-0- $\beta$-D-galactopyranosyl-D-glucose. J. Dairy Sc., 61, 33-38.

[11] WebB (B. H.) and Johnson (A. H.) (1965). - Fundamentals of Dairy Chemistry. The AVI publishing company, Westport, Connecticut, 17 and 19. 